Ann. Biol. anim. Bioch. Biophys., I970, 10 (I), I7I-I74.

NOTE

\title{
INFLUENGE DE LA CONSERVATION SUR LA VALEUR NUTRITIVE DES PROTÉINES DU BLÉ
}

\author{
M. PAWLAK et R. PION
}

Station d'Études des Métabolismes, Centre de Recherches de Clermont-Ferrand, 63 -Saint-Genès-Champanelle

Institut national de la Recherche agronomique

Nous avions constaté, au cours de l'étude de l'influence de la teneur en lysine de la ration sur les concentrations de cet acide aminé à l'état libre, dans le sang et le muscle du Rat en croissance (PAWLAK et PION, I968 a) que les croissances observées pour des régimes de composition similaire étaient plus faibles dans certaines expériences que dans d'autres. Or, les croissances les plus faibles étaient celles des animaux consommant des blés conservés au moins un an, et il était permis de penser que les différences observées pouvaient être dues, au moins en partie, à une modification du blé au cours de la conservation. Aussi avons-nous étudié l'influence du temps et des conditions de conservation sur la valeur nutritive du blé.

Les expériences ont consisté à mesurer les croissances obtenues par les rats consommant les différents blés et à déterminer en fin d'expérience les teneurs en acides aminés libres des tissus sanguin et musculaire, afin de comparer ces dernières avec celles que nous avons observées précédemment et en particulier aux courbes de références correspondant à des apports croissants de lysine et de thréonine chez les animaux dont tous les autres besoins sont satisfaits à l'exception de l'acide aminé étudié (PAWLAK, PIon, I968 $a, b$ ). Cette comparaison nous permet d'apprécier l'état de nutrition azotée des animaux et la disponibilité de la lysine et de la thréonine des différents blés étudiés, compte tenu des teneurs en acides aminés de ces blés et des quantités retrouvées au niveau des tissus.

Les essais concernant les durées de conservation ont été effectués avec un blé Aronde de la récolte 1966 ayant subi des durées de conservation de 6 mois, I 2 mois, I 5 mois, 2 r mois et 27 mois dans des conditions normales de stockage. Nous avons également comparé le blé $A$ yonde de 2 I mois avec un blé stocké depuis 4 ans dans des conditions normales.

L'influence du mode de stockage a été étudié sur des blés Capelle conservés 2 ans sous atmosphère de gaz carbonique, d'azote ou ventilés, et un témoin conservé à $-20^{\circ}$.

Les animaux utilisés dans ces expériences sont des rats mâles de souche Wistar, répartis en lots de 8 , élevés en cages individuelles et dont les consommations et les gains de poids sont contrôlés. Ces animaux reçoivent à volonté les régimes expérimentaux à partir du poids de I Io g jusqu'au poids de $\mathrm{I} 8 \mathrm{o} \mathrm{g}$ (soit pendant une durée moyenne de $\mathrm{r}_{4}$ jours).

Les régimes sont constitués de façon à satisfaire tous les besoins des animaux et en particulier 
les besoins en acides aminés, dans l'hypothèse où ceux-ci sont parfaitement disponibles. Le blé est supplémenté en DL-méthionine, L-Tryptophane, L-Thréonine et L-lysine et additionné de minéraux et vitamines : l'énergie brute est maintenue à $45^{\circ 0} \mathrm{kcal} / \mathrm{kg}$ de MS et le taux protéique à $\mathrm{I} 2 \mathrm{p}$. IOO.

En fin de période expérimentale, le sang et les muscles des membres postérieurs sont prélevés ; les acides aminés libres en sont extraits respectivement par l'éthanol à $84 \mathrm{p}$. Ioo et l'acide picrique I p. Ioo puis dosés par chromatographie sur colonne à l'aide d'un appareil automatique.

Les gains de poids des animaux (tabl. 1) nourris avec les blés conservés de 12 mois à 26 mois sont inférieurs en moyenne de 20 p. Ioo à ceux obtenus après l'ingestion du blé frais ( 6 mois) sans que l'on puisse noter de différence importante entre eux. Cette réduction de gain de poids est liée à une diminution de la consommation de 15 p. Ioo.

TABLEAU I

Expérience blé Aronde récolte 1966

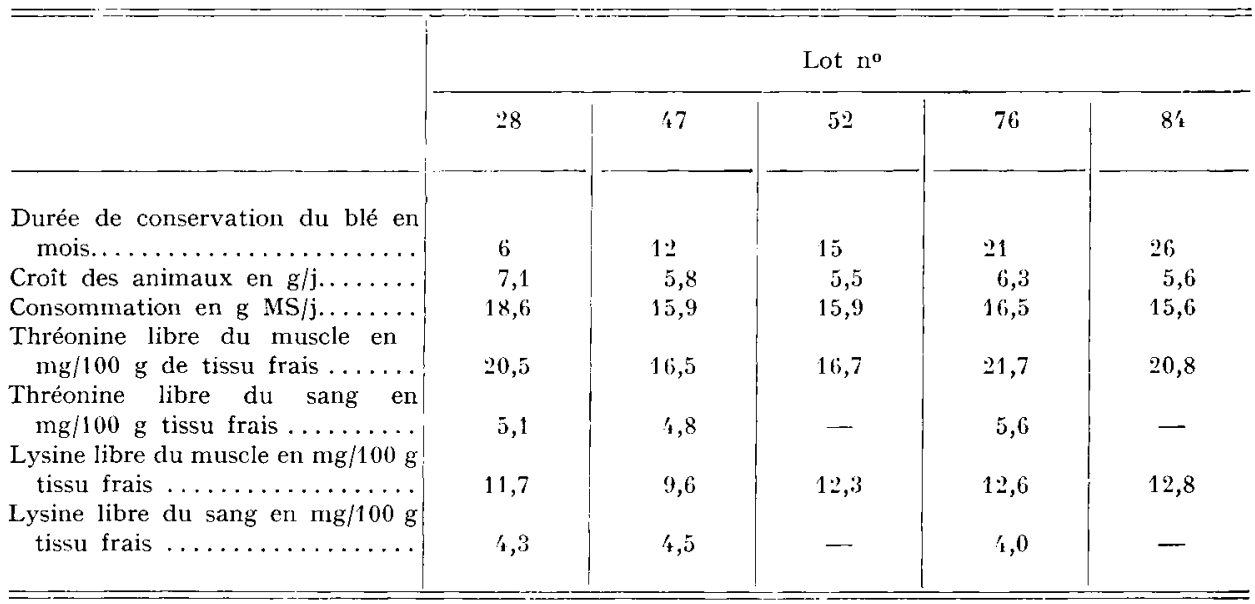

De même, le gain de poids journalier des animaux consommant du blé conservé 4 ans ( $5, \mathrm{r} \mathrm{g} /$ jour) est plus faible que celui des animaux ingérant le blé conservé 2 ans $(6,3 \mathrm{~g} /$ jour$)$, soit une réduction de 20 p. Ioo alors que la quantité de MS ingérée par jour n'est réduite que de Io p. Ioo (tabl. 2).

Les blés conservés sous atmosphère d'azote ou de gaz carbonique, ou ventilés et le témoin conservé à $-20^{\circ}$, ont été comparés entre eux et avec un blé Aronde de 2 ans. Les gains de poids des animaux, ingérant les régimes à base de blé ayant subi les trois traitements différents, ou les deux régimes servant de témoins sont voisins à l'exception du lot ventilé pour lequel la croissance a été légèrement plus faible. Les consommations journalières des différents lots sont également voisines (tabl. 3 ).

Les teneurs en acides aminés libres observées dans les tissus sanguin et musculaire des animaux consommant les régimes à base de blé Aronde stockés de 6 à 26 mois dans des conditions normales sont voisines. Les teneurs en lysine et thréonine libre du sang et du muscle (tabl. r) correspondent aux teneurs observées dans les expériences précédentes, pour les mêmes apports de lysine et de thréonine des régimes. Les besoins des animaux en ces deux acides aminés semblent donc satisfaits, et la conservation du blé pendant 2 ans ne semble pas entraîner d'indisponibilité de la lysine et de la thréonine.

Il ne semble pas, d'après les résultats obtenus, que les croissances inférieures à l'optimum observé chez les animaux consommant des blés stockés plus d'un an dans diverses conditions soient, à une exception près, liées à une indisponibilité de la lysine ou de la thréonine. Elles peuvent 
être dues à d'autres nutriments que les protéines ou à l'indisponibilité d'autres acides aminés. Toutefois, la supplémentation du blé conservé 4 ans par de la valine, et de l'isoleucine et par une quantité plus importante de méthionine, ne modifie ni la consommation ni la croissance des rats.

TABIEAU 2

Influence de la conservation de longue durée

\begin{tabular}{|c|c|c|}
\hline & \multicolumn{2}{|c|}{ Lot $n^{\circ}$} \\
\hline & 76 & 77 \\
\hline Durée de conservation.............. & 21 mois & 4 ans \\
\hline Croit des animaux en $\mathrm{g} / \mathrm{j} \ldots \ldots \ldots$ & 6,3 & 5,1 \\
\hline Consommation en $\mathrm{g} \mathrm{MS} / \mathrm{j} \ldots \ldots \ldots \ldots$ & 16,5 & 15,1 \\
\hline 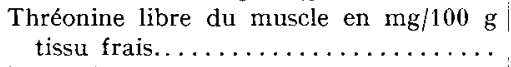 & 21,7 & 23,6 \\
\hline 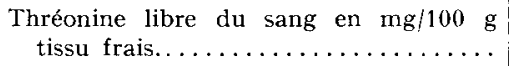 & 5,6 & 5,2 \\
\hline 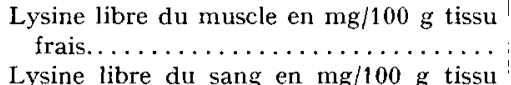 & 12,6 & 16,9 \\
\hline 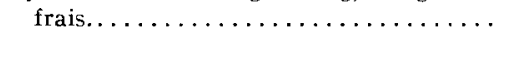 & 5,0 & 5,3 \\
\hline
\end{tabular}

TABLEAU 3

Influence des conditions de conservation

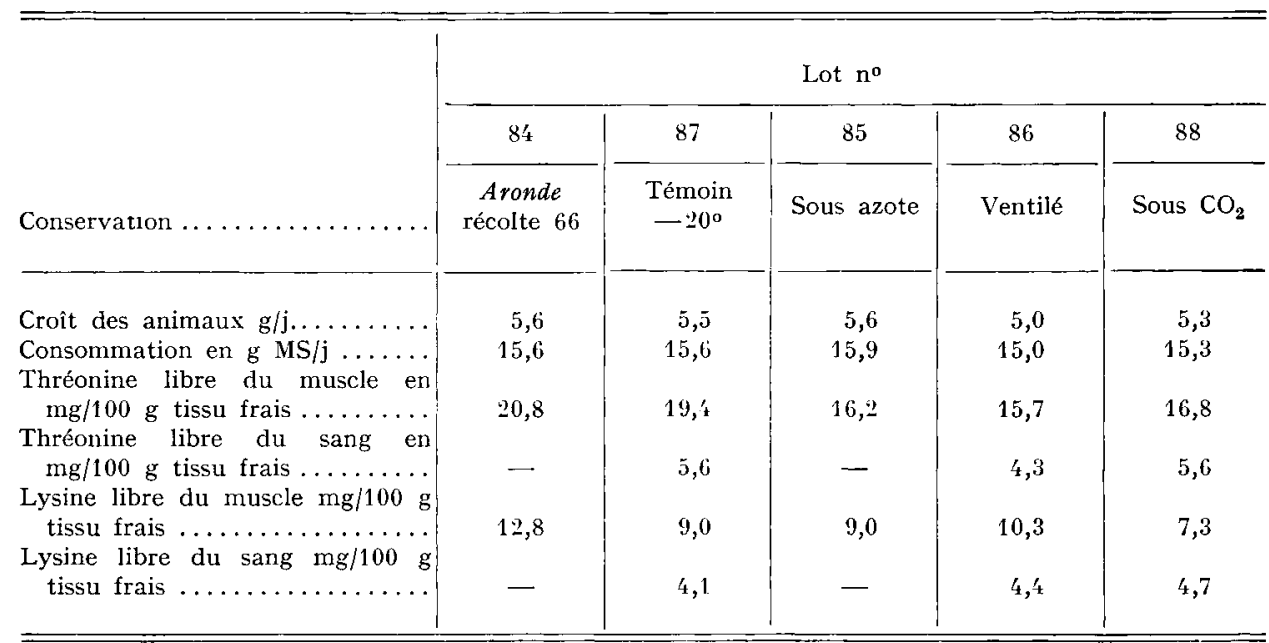

Les tissus des animaux consommant le blé ventilé contiennent relativement peu de thréonine libre, alors que leurs teneurs en lysine sont normales. La teneur en lysine libre des muscles des animaux consommant le blé conservé sous gaz carbonique est également un peu faible. Les valeurs observées pour les autres conditions de conservation sont normales. 


\section{SUMMARY}

EFFECT OF STORAGE ON THE FEEDING VALUE OF WHEAT PROTEINS

Several groups of growing rats were given balanced diets with wheat supplemented by DLmethionine, L-lysine, L-tryptophan and L-threonine, and added minerals and vitamins. The wheat samples had been stored either under standard conditions for $6,12,15,21,26$ months or 4 years, or under controlled atmosphere during 2 years.

Growth rates and food consumptions were recorded, and free amino acid contents were estimated in blood and muscle at the end of the experiments.

A I year or longer storage time (tables I and 2) resulted in a slight decrease in growth rates and food consumptions, without any change in lysine and threonine contents of blood and muscle. The wheat samples stored under nitrogen or $\mathrm{CO}_{2}$ atmosphere and the control stored at $-20^{\circ} \mathrm{C}$ gave similar results. Growth rates and food consumption were the lowest with the air-stored wheat sample (table 3 ).

\section{RÉFÉRENCES BIBLIOGRAPHIQUES}

Pawlak M., Pion R., Ig68 a. Influence de la supplémentation des protéines de blé par des doses croissantes de lysine sur la teneur en acides aminés libres du sang et du muscle du rat en croissance. Ann. Biol. anim. Biochim. Biophys., 8, 517-530.

Pawlak M., Pion R., I 968 b. Influence de la supplémentation des protéines du blé par des doses croissantes de thréonine sur les teneurs en acides aminés libres du sang total et du muscle du rat en croissance. C. R. Acad. Sci. Paris, série D, 266, I993-1995. 